\title{
Gap opening in topological-defect lattices in graphene
}

\author{
Joice da Silva-Araújo, H. Chacham, and R. W. Nunes* \\ Departamento de Física, ICEX, Universidade Federal de Minas Gerais, \\ CP 702, 30123-970,Belo Horizonte, MG, Brazil.
}

(Dated: March 3, 2022)

\begin{abstract}
Ab initio calculations indicate that topological-defect networks in graphene display the full variety of single-particle electronic structures, including Dirac-fermion null-gap semiconductors, as well as metallic and semiconducting systems of very low formation energies with respect to a pristine graphene sheet. Corrugation induced by the topological defects further reduces the energy and tends to reduce the density of states at the Fermi level, to widen the gaps, or even to lead to gap opening in some cases where the parent planar geometry is metallic.

PACS numbers: 73.22.-f, 73.20.Hb, 71.55.-i
\end{abstract}


Since its recent experimental discovery ${ }^{\underline{1}}$, graphene has emerged as an unique condensedmatter system, for its remarkable electronic and electromechanical properties, as well as a test-bed for relativistic-dynamics phenomena, due to the linear and isotropic dispersion of the electronic states near the Fermi level, that leads to the massless-Dirac-fermion nature of its low-energy electronic excitations ${ }^{2}$. Devising gap-opening and gap-control mechanisms is one of the key issues related to the prospective application of graphene as the new holy-grail of electronic devices $2, \underline{3}$. Several mechanisms for changing, perhaps tailoring, the electronic structure of graphene have been proposed. Experimental and theoretical works have indicated the occurrence of gap opening at the Dirac point in graphene due to interaction with insulating substrates ${ }^{4}$. The generation of additional Dirac cones of massless fermions has also been identified, due either to imposed periodic perturbations ${ }^{5}$, or associated with a periodic pentagon-heptagon superstructure on grain boundaries in polycrystalline graphene ${ }^{6}$. Of no smaller relevance are the effects of corrugation on the thermodynamic stability and transport properties of substrate-supported and free-standing graphene ${ }^{2,7-10}$. What causes corrugation in graphene is an open issue: the possibility of corrugation being an intrinsic feature of the material ${ }^{8}, \underline{9}$, due to the interaction with a substrate $\frac{10}{}$, or the effect of adsorbates 11 , are scenarios that have been put forward in the literature.

In this work, we employ ab initio calculations to examine the possibility of topological curvature, its stability and energetics, and its effects on the electronic structure of graphene sheets. Topological curvature fields are generated when, following the formation of a StoneWales defect $(\mathrm{SW})$ by a $90^{\circ}$ rotation of a carbon-carbon bond ${ }^{12}$, the two pentagons and two heptagons comprising the SW are separated, and the defect is fully dissociated. We consider periodic lattices containing two pentagons and two heptagons, such that these isolated odd-membered rings develop local curvature fields, while preserving the null average curvature of the parent graphene sheet. Our study broadens the scope of the pentaheptite allotrope of carbon previously introduced by Crespi and collaborators $\frac{12}{2}$, that consisted of a two-dimensional (2D) carbon sheet composed entirely of pentagons and heptagons. Our results raise the possibility of the generation of semiconducting and metallic graphene sheets by the introduction of topological defects (TD). We find that periodic networks of TDs may present the full variety of electronic structures, at the single-particle level, including genuine metals, "graphene-like" null-gap semiconductors, and also finite-gap ( 0.02-0.75 eV) semiconductors. A remarkable result of our calculations is the identification of metallic and 
semiconducting structures with very small formation energies $(\sim 0.1 \mathrm{eV} /$ atom with respect to graphene), in which the corrugation induced at the TD sites plays a key role in reducing the energy. Being a full first principles address of fully dissociated TDs, our work differs from previous theoretical works on corrugated graphene and on topological defects in this material13,14. In particular, our results cast doubt on the assumption that Dirac points are robust against the introduction of a distribution of TDs in graphene $\frac{14}{}$.

Our calculations are performed in the framework of Kohn-Sham density functional theory (DFT), within the generalized-gradient approximation (GGA) $\stackrel{15}{\underline{m}}$ and norm-conserving pseudopotentials in the Kleinman-Bylander factorized form $\stackrel{16}{ }$. We use the LCAO method implemented in the SIESTA code $\frac{17}{}$, with a double-zeta basis set plus polarization orbitals. Calculational parameters are set such that total-energy differences are converged to better than $1 \mathrm{meV} /$ atom, with residual atomic forces no larger than $0.02 \mathrm{eV} / \AA$. Single-particle energy eigenvalues are converged to $\sim 1 \mathrm{meV}$.

The pentaheptite form of graphene investigated by Crespi et al. $\frac{12}{1}$ is shown in Fig. 1(a). The structures we investigate in this work, which can be considered a generalization of the pentaheptite, are generated from three different seed structures, as shown in Fig. 1. The first seed in Fig. 1(a) is the pentaheptite itself, with eight atoms in the unit cell. The second seed, in Fig. 1(b), has a 16-atom unit cell with a SW surrounded by hexagons. The third seed, shown in Fig. 1(c), has a 24-atom unit cell where the pentagons and heptagons are already dissociated, each being fully surrounded by one shell of hexagons. Larger unit cells can be constructed from the seeds, by introducing shells of hexagons surrounding the TDs. In order to establish a nomenclature, let the 8-atom seed be called $S_{11}$, with a TD number density $n(1,1)=0.18$ (in units of $\AA^{-2}$ ). The 16 -atom seed is then labeled $S_{21}$, and the 24-atom seed is $S_{31}$. From these we can generally build an $S_{i j}$ geometry which is the $j$-th member of the $i$-th family, having an $8 \times i \times j^{2}$-atom unit cell and a TD number density of $n(i, j)=n(1,1) /\left(i \times j^{2}\right)$. Here, we consider a total of nine planar geometries, of which: four are generated from the 8-atom seed, namely $S_{11}, S_{12}, S_{13}$, and $S_{14}$; three are built from the 16-atom seed, namely $S_{21}, S_{22}$, and $S_{23}$; and two from the 24-atom seed, namely $S_{31}$ and $S_{32} \underline{18}$.

For all but the $S_{11}$ and $S_{21}$ geometries, the SW defects are fully dissociated, and corrugated structures can be generated by allowing for the local curvature fields generated by the isolated TDs to develop. Thus, a total of 16 structures were considered, nine of which 
are planar and seven are corrugated. Figure 2 shows a 2x2 unit cell of the planar and the corrugated $S_{23}$ geometries. In all cases, the planar geometries did not develop corrugation spontaneously under structural relaxation. In order to investigate whether this was an indication of metastability of the planar forms, we built slightly corrugated initial geometries with random off-plane shifts of the atoms imposed on the planar structures. Upon structural optimization the $S_{31}[n(3,1)=0.060], S_{12}[n(1,2)=0.045]$, and $S_{22}[n(2,2)=0.023]$ geometries relaxed back to the planar form for off-plane shifts of $0.01 \AA$, and developed corrugation for larger shifts, with the exception of the $S_{31}$, with a higher TD concentration, that only developed corrugation for shifts of $0.1 \AA$ or larger. Thus, we find numerical evidence for a metastability of the planar structures with high TD concentrations, down to a critical density of TDs $\left(n_{c} \sim 0.023\right)$, below which a corrugation instability sets in, as indicated in Fig. 3. The instability is related to the fact that, for the more diluted structures, large incipient conical and saddle-like regions are associated with the isolated pentagons and heptagons, respectively, and the cost of flattening such regions onto the plane becomes correspondingly larger $\underline{19}$.

In Fig. 3 we show, for the 16 structures, the formation energy (with respect to pristine graphene) $E_{f}=E_{t o t} / N-E_{\text {graph }}$ (in eV/atom) as a function of the TD number density, where $N$ is the number of atoms in the unit cell, $E_{\text {tot }}$ is the the total energy per unit cell, and $E_{\text {graph }}$ is the total energy per atom for a pristine graphene sheet. Since the planar sheets remained numerically stable, we include in the figure the energy of their "relaxed" geometries. Clearly, dissociation of TDs, when constrained to the plane, incurs an energy cost that behaves linearly with $n$ (with a negative slope), with the exception of the $S_{14}$, a structure in the limit of the connectivity of such geometries in the plane ${ }^{18}$. Corrugation leads to a reduction of the formation energy that is comparable to or larger than the formation energy itself for all but the $S_{31}$ and $S_{12}$ geometries. We define a corrugation energy by the difference between the formation energies of the planar and corrugated geometries $E_{c}=E_{f}^{\text {plane }}-E_{f}^{\text {corr }} . E_{f}$ and $E_{c}$ values are included in Table I. Note the correlation between $E_{f}$ of the planar geometries and $E_{c}$ in Fig. 3, the more diluted structures have large $E_{f}$ values in the planar form and also large values of $E_{c}$, leading to a trend, for the corrugated geometries in each family, of decreasing formation energies as the TD concentration decreases.

Of note also is the fact that the $S_{2 j}$ family is a low energy branch in Fig. 3, both for planar and corrugated forms, while the $S_{1 j}$ and $S_{3 j}$ families have comparable energies. Among the 
seed geometries, the $S_{21}$ has a much lower formation energy than the $S_{11}$ and $S_{31}$. This can be understood by comparing the seed structures: the $S_{11}$ seed has zero hexagons and four TDs in the unit cell; the $S_{21}$ has four hexagons and four TDs, and the the $S_{31}$ has eight hexagons and four TDs. In the latter, the hexagons are highly strained due to the SW dissociation, and this structure has the highest $E_{f}$ among the seeds. The $S_{21}$ has four slightly strained hexagons in its periodic unit, and this considerably lowers its energy when compared with the $S_{11}$ and $S_{31}$. As a result of the balance between hexagons and TDs in the seed, the bond-stretching and bond-bending distortions in the $S_{2 j}$ family are smaller than in the other two families. To quantify these structural features, we compute the Keating-model elastic energies of the 16 structures. The results are included in Fig. 3 and in Table I. Note that the $a b$ initio $E_{f}$ and the Keating energies follow the same overall trends, with the $S_{2 j}$ family appearing as a low-elastic-energy branch in Fig. 3 .

Also related to these structural features of the seeds is the degree of corrugation of each structure. We define the atomic corrugation $Z_{c}$ of the non-planar structures as the distance of the center of the protruding pentagons to the average plane. The values of $Z_{c}$ are given in Table I, and the inset in Fig. 33 shows the behavior of $Z_{c}$ as a function of $n$. Note that the $S_{2 j}$ family represents a low corrugation branch, due to its comparably less strained planar geometry than the corresponding ones in the $S_{1 j}$ and $S_{3 j}$ families. This also explains why the planar $S_{22}$ with $n(2,2)=0.023$ is metastable, while the the planar $S_{13}$ with $n(1,3)=0.020$ is unstable against corrugation.

The diverse nature of the electronic states of these TD periodic lattices can be anticipated by examining the DOS plots of the seed structures in Fig. 1. The DOS for the $S_{11}$ seed, in Fig. 1(d), displays a metallic character, with an ab initio value for the DOS at the Fermi level that is half of the tight-binding result in Ref. 12. The $S_{21}$ seed, in Fig. 1(e), shows a "graphene-like" Dirac-fermion DOS that derives from linear electronic bands crossing each other at the Fermi point, forming a Dirac cone on a much reduced energy scale $(\sim[-$ $0.1,0.1 \mathrm{eV}$ ), beyond which other bands intervene and break the electron-hole symmetry. The $S_{31}$ seed is a semiconductor [DOS is shown in (f)] with a GGA-DFT gap of $\sim 0.6 \mathrm{eV}$.

Regarding the general aspects of the electronic structure of all planar and corrugated structures in our study, a first observation concerns the role played by topology, relaxation, and corrugation, in determining the nature of the electronic states. In order to address the effects solely of the topological transformation (TT), we compute the DOS and the electronic 
TABLE I: Physical properties of TD networks in graphene. Formation energy $E_{f}$, corrugation energy $E_{c}$ (see text), and elastic energies $E_{e l}$. are in units of $\mathrm{eV} / \AA . Z_{c}$ (in $\AA$ ) is the measure of corrugation (see text). $E_{g}$ (in $\mathrm{eV}$ ) is the energy gap for the semiconducting systems. The value of DOS at the Fermi level (in number of states per energy per atom) is given for the metallic systems. The accumulated charge on the pentagons $Q_{P}$ is given for the corrugated geometries.

\begin{tabular}{|c|c|c|c|c|c|c|c|c|c|c|}
\hline & \multicolumn{5}{|c|}{ planar } & \multicolumn{7}{|c|}{ corrugated } \\
\hline$S_{i j}$ & $E_{f}$ & $E_{e l .}$ & $E_{g}$ & DOS & $E_{f}$ & $E_{c}$ & $Z_{c}$ & $E_{g}$ & DOS & $Q_{P}$ \\
\hline$S_{11}$ & 0.24 & 0.32 & - & 0.05 & - & - & - & - & - & - \\
\hline$S_{12}$ & 0.40 & 0.38 & - & 0.07 & 0.35 & 0.05 & 1.03 & - & 0.04 & -0.016 \\
\hline$S_{13}$ & 0.44 & 0.42 & 0.53 & - & 0.21 & 0.23 & 2.58 & 0.67 & - & -0.015 \\
\hline$S_{14}$ & 0.46 & 0.44 & 0.02 & - & 0.15 & 0.31 & 4.03 & - & 0.02 & -0.015 \\
\hline$S_{21}$ & 0.14 & 0.18 & 0.00 & 0.00 & - & - & - & - & - & - \\
\hline$S_{22}$ & 0.26 & 0.24 & - & 0.03 & 0.16 & 0.10 & 1.80 & 0.03 & - & -0.009 \\
\hline$S_{23}$ & 0.29 & 0.26 & 0.42 & - & 0.09 & 0.20 & 3.29 & 0.39 & - & -0.012 \\
\hline$S_{31}$ & 0.36 & 0.37 & 0.61 & - & 0.33 & 0.03 & 0.58 & 0.74 & - & 0.000 \\
\hline$S_{32}$ & 0.47 & 0.44 & 0.00 & 0.00 & 0.17 & 0.30 & 3.28 & 0.50 & - & -0.013 \\
\hline
\end{tabular}

bands of the (planar) seed structures in three different situations, as also shown in Fig. 1: (1) a structure with only the TT imposed on graphene, without any relaxation of either atomic positions or lattice vectors; (2) with the TT and relaxation of atomic positions only; (3) and finally, with the TT and also relaxation of the lattice vectors and atomic positions. For the $S_{11}$, in Fig. 1(d), relaxation does not bring any significant changes, and the system is metallic in the three cases, with little change in the position of the Fermi level. Things are quite different for the $S_{21}$, in Fig. 1(e), which is a metallic system in the first two scenarios above, but develops a Dirac-fermion-like band structure when lattice relaxation is allowed, with the Fermi level lying on the Dirac point. For the $S_{31}$ we only computed the electronic states for the cases (1) and (3). The DOS's are shown in Fig. 1(f), where case (1) is seen to behave as a metal, with the Fermi level lying at the bottom part of a set of bands that start above an energy gap of about $0.5 \mathrm{eV}$. After full relaxation, enough spectral weight is shifted to lower energies for the Fermi level to lye on the gap, and the system to become a semiconductor. Hence, the TT by itself does not explain the behavior of the electronic states we observe in our calculations, with relaxation effects playing a crucial role. The realistic 
treatment of the interatomic interactions, provided by the ab initio method, is thus a key ingredient.

In Table I we include the values of the gap for all semiconducting TD networks, and the value of the DOS at the Fermi level for metallic ones. Generally, corrugation tends to reduce the DOS at the Fermi level, to widen the gap, or even lead to gap opening in some cases where the parent planar geometry is metallic. There is one exception, however, in the case of the $S_{14}$ which displays a small gap $(0.02 \mathrm{eV})$ in the planar form and is a metal in the corrugated geometry. Overall, a total of nine structures are semiconductors, seven of which with gaps ranging from 0.39 to $0.74 \mathrm{eV}$. Five of the metastable systems in our study have formation energies below $0.2 \mathrm{eV} /$ atom $\left(E_{f}=0.39 \mathrm{eV} /\right.$ atom for a fullerene molecule with the same methodology). Among these, the planar $S_{21}$ is a Dirac-fermion null-gap semiconductor, the corrugated $S_{14}$ is a metal, and the corrugated $S_{22}, S_{23}$, and $S_{32}$ are semiconductors. The corrugated $S_{23}$ TD network stands out as a semiconductor with very small formation energy (0.09 eV/atom) for a carbon system.

The low formation energies of the corrugated TD networks in our study, as well as the $Z_{c}$ values which are in the range of the corrugation observed experimentally $\underline{\underline{2}, \underline{7}-\underline{10}}$, suggest that consideration should be given to these structures as a model for the rippled graphene samples in the experiments. A feature of corrugated graphene that has been experimentally observed is the formation of electron-hole puddles, with a concentration of electrons in the higher parts of the samples ${ }^{20}$. In our corrugated geometries we find that the pentagons at the higher parts of the ripples are electron-rich (except for the corrugated $S_{31}$ ) as shown in Table I. Moreover, the low formation energies indicate that the synthesis of such structures may be feasible. We anticipate that kinetics would be the only potentially limiting factor, since besides the low formation energies, the barriers protecting such structures from reverting back to pristine graphene should be of the order of the $7 \mathrm{eV}$ estimate for the $S_{11}$ in Ref. 12 . Regarding a pressure-driven transformation of graphite into a three-dimensional bulk of TD networks, as envisaged in Ref. 12, we note that the areal density of all corrugated structures in our study is larger than that of graphene, e.g., one carbon atom per $2.35 \AA^{2}$ for the $S_{23}$ compared with one carbon atom per $2.69 \AA^{2}$ for graphene, which would favor a pressuredriven transformation. We add that very recent experiments have indicated the formation of both clustered and isolated pentagon-heptagon pairs in graphene samples obtained by reduction of graphene-oxide ${ }^{21}$, and corrugation was observed in the areas of the samples 
containing the defects. Further refinement of such reduction techniques may lead to a viable synthesis routes for TD networks. Moreover, this also suggests that other chemistry-based synthesis techniques may also be possible $\stackrel{12}{2}$.

In summary, ab initio calculations indicate that the electronic structure of topologicaldefect networks in graphene range from Dirac-fermion null-gap semiconductors to finitegap semiconductors and metals, some of them with very low formation energies, offering a possible route for tailoring the electronic nature of graphene sheets. Corrugation induced by the topological defects further reduces the energy and modifies the electronic structure of the sheets.

\section{Acknowledgments}

We thank José M. Soler for useful discussions, and acknowledge support from CNPq, FAPEMIG, and Instituto do Milênio em Nanociências-MCT, Brazil.

* corresponding author; Electronic address: rwnunes@fisica.ufmg.br

1 K. S. Novoselov et al., Science 306, 666 (2004).

2 A. H. Castro Neto et al., Rev. Mod. Phys. 81, 109 (2009), and references therein.

3 J. B. Oostinga et al., Nature Mater. 7, 151 (2008).

4 S. Y. Zhou et al., Nature Mater. 6, 770 (2007); S. Kim, J. Ihm, H. J. Choi, and Y. -W. Son, Phys. Rev. Lett. 100, 176802 (2008); G. Giovannetti et al., Phys. Rev. B 76, 073103 (2007).

5 C. -H. Park et al., Phys. Rev. Lett. 101, 126804 (2008); I. Pletikosić et al., Phys. Rev. Lett. 102, 056808 (2009); S. S. Carara et al., Phys. Rev. B 80, 115435 (2009).

6 J. daSilvaAraújo and R. W. Nunes, Phys. Rev. B 81, 073408 (2010).

7 C. H. Lui et al., Nature 462339 (2009).

8 J. C. Meyer et al., Nature 446, 60 (2007).

9 A. Fasolino, J. H. Los, M. I. Katsnelson, Nature Mater. 6, 858 (2007).

10 F. Varchon, P. Mallet, J. Y. Veuillen, and L. Magaud, Phys. Rev. B 77, 235412 (2008).

11 R. C. Thompson-Flagg, M. J. B. Moura, and M. Marder, EPL 85, 46002 (2009).

12 V. H. Crespi, L. X. Benedict, M. L. Cohen, and S. G. Louie, Phys. Rev. B 53, R13303 (1996). 
FIG. 1: (Color online) (a-c): $2 \times 2$ unit cells of seed structures. (a) $S_{11}$, (b) $S_{21}$, and (c) $S_{31}$. Primitive-cell vectors are indicated. (d-f): DOS for the structures (a) to (c). The DOS's are depicted for the structures with only the topological transformation (dashed red lines), with relaxation of atomic positions (dot-dashed blue lines), and with relaxation of atomic positions and lattice vectors (full black lines).

FIG. 2: (Color online) (a) Planar and (b) corrugated forms of the $S_{23}$, the lowest-energy topologicaldefect network.

13 F. Guinea, M. I. Katsnelson, and M. A. H. Vozmediano, Phys. Rev. B 77, 075422 (2008).

14 A. Cortijo and M. A. H. Vozmediano, Phys. Rev. B 79, 184205 (2009); Nucl. Phys. B 763, 293 (2007).

15 W. Kohn and L. J. Sham, Phys. Rev. 140, A1133 (1965); J. P. Perdew, K. Burke, and M. Ernzerhof, Phys. Rev. Lett. 773865 (1996).

16 N. Troullier and J .L. Martins, Phys. Rev. B 43, 1993 (1991); L. Kleinman and D. M. Bylander, Phys. Rev. Lett. 48, 1425 (1982).

17 J. M. Soler et al., J. Phys. Cond. Matt. 142745 (2002).

18 For structures with smaller TD densities and thus larger incipient conical and saddle-like regions, it is not possible to build a fully threefold-connected network, with reasonable bond-length values, without introducing corrugation. In practice, we found this to be true already for a planar $S_{33}$ structure, with $n(3,3)=0.007$, for which we were not able to seam the tears that open when additional shells of hexagons are introduced between the TDs.

19 In Ref. 12 it was suggested that sufficiently homogeneous distributions of equal numbers of pentagons and heptagons should be stable in the planar form. Our calculations indicate that, generally, too dilute distributions $\left(n<n_{c}\right)$ should be unstable against corrugation.

20 A. L. Vázquez de Parga et al., Phys. Rev. Lett. 100, 056807 (2008).

21 C. Gómez-Navarro et al., Nano Lett. 10, doi:10.1021/n19031617 (2010). 
FIG. 3: (Color online) Ab initio formation energy of planar (filled) and corrugated (empty symbols) TD networks, as a function of TD density. Symbols with a diagonal stripe filling show the Keatingmodel elastic energies of the planar geometries. Planar structures to the right of the dashed vertical line are metastable. Inset shows corrugation height as a function of TD density. Lines are guides to the eye. 